\title{
The Variants Within the COL5A1 Gene are Associated with Reduced Risk of Anterior Cruciate Ligament Injury in Skiers
}

\author{
by \\ Marta Stęień-Słodkowska ${ }^{1}$,Krzysztof Ficek ${ }^{1}$, Mariusz Kaczmarczyk², \\ Agnieszka Maciejewska-Karłowska1, Marek Sawczuk , Agata Leońska-Duniec ${ }^{1,2}$, \\ Miłosz Stẹpiński, Paweł Ziętek ${ }^{3}$, Paweł Król ${ }^{4}$, Monika Chudecka ${ }^{1}$, \\ Pawet Cięszczyk $k^{1,2}$
}

The purpose of this study was to examine the association of the BstUI RFLP C/T (rs 12722) and DpnII RFLP C/T (rs 13946) COL5A1 polymorphisms, individually and as haplotypes, with anterior cruciate ligament ruptures in recreational skiers. Subjects were 138 male recreational skiers with surgically diagnosed primary anterior cruciate ligament ruptures. The control group consisted of 183 apparently healthy male recreational skiers, who were without any self-reported history of ligament or tendon injury. DNA was extracted from buccal cells donated by the subjects and genotyping was carried out using real-time PCR. The genotype distributions for both polymorphisms met HardyWeinberg expectations in both groups. There were no significant differences in genotype distribution of allele frequencies of COL5A1 BstUI RFLP C/T and COL5A1 DpnII RFLP C/T polymorphisms between the ACL rupture and control groups. The T-T (BstUI RFLP T, DpnII RFLP T) haplotype was the most common (55.6\%). The haplotype $T-C$ was not present in any of the subjects. There was an underrepresentation tendency of the $C-T$ haplotype in the study group compared to controls under recessive mode of inheritance. Higher frequency of the COL5A1 BstUI RFLP C/T and COL5A1DpnII RFLP C/T polymorphisms haplotype is associated with reduced risk of anterior cruciate ligament injury in a group of apparently healthy male recreational skiers.

Key words: ACL rupture, collagen, polymorphisms, real-time polymerase chain reaction.

\section{Introduction}

Skiing as a recreational activity has gained a lot of interest in the last twenty years ( $\mathrm{O}^{\prime}$ Donnel et al., 2007). Injuries in alpine skiing have been a serious concern since the very beginning of the sport (Spörri et al., 2012). The injury pattern has changed over this time period, due to alterations and improvements in ski equipment ( $\mathrm{O}^{\prime}$ Donnel et al., 2007), however, the risk of injury is still high. During a single skiing season throughout Switzerland, around 1000 alpine skiers and snowboarders per day were diagnosed with injuries serious enough to require medical treatment (Hasler et al., 2011). The knees are most commonly affected. Injuries to the anterior cruciate ligament of the knee are common in populations with regular physical activity and may seriously influence the risk of early-onset posttraumatic osteoarthritis regardless of the treatment administered (Smith et al., 2011). Recent developments that enhance understanding of the

1 - University of Szczecin, Department of Physical Culture and Health Promotion, Szczecin, Poland.

2 - Gdansk University of Physical Education and Sport, Faculty of Tourism and Recreation, Gdansk, Poland.

3 - Medical University at Szczecin, Department of Orthopedics and Traumatology of Pomeranian Medical, Szczecin, Poland.

4. University of Rzeszow, Department of Physical Culture, Rzeszow, Poland. 
role of individual genes in human body's processes should contribute to injury prevention as well as sport rehabilitation. Thus, the role of genetics in sport research increases each year (Sawczuk et al., 2011).

The knee joint comprises a number of tissues: bones, muscle, tendons and ligaments, among others (Boyan et al., 2013). It relies on other structures to provide both static and dynamic stability: the anterior and posterior cruciate ligaments, the medial and lateral collateral ligaments, the menisci, the capsule and the muscles crossing the joint (Frizziero et al., 2013). Knee stability is largely dependent on the ability of the anterior cruciate ligament (ACL) to resist anterior tibial translational loads and rotational loads (Dhillon et al., 2011).

Most ACL injuries in sports occur during a non-contact episode, typically during deceleration, lateral pivoting, or landing tasks that are associated with high loads on the knee joint. Therefore, sports maneuvers may lead to high knee loads (Myer et al., 2011). The most frequent "slip-catch" mechanism of ACL injury was identified in the World Cup alpine skiing. The injury mechanism is characterized by a common pattern in which the inside edge of the outer ski catches the snow surface while turning, forcing the knee into valgus and tibial internal rotation (Bere et al., 2013). However, numerous studies have shown that ACL ruptures are complex, multifactorial and determined by the interaction of extrinsic and intrinsic risk factors (Hewett et al., 2010).

The ACL is composed of dense fibrous connective tissue that attaches the femur to the tibia. It has a large amount of collagen fibers arranged in a hierarchal pattern giving it high tensile strength (Tovar et al., 2010). Collagen is the main component of ligaments $\left(\mathrm{O}^{\prime}\right.$ Connell et al., 2013) and its fibrils may be composed of collagen type I, III, VI, V, XI and XIV (Frank et al., 2004). Type $\mathrm{V}$ collagen is a quantitatively minor fibrillar collagen, but most prominent in connective tissue where it plays an important role in regulating fiber diameter, initiating fibril assembly and regulating lateral fibril growth within tendons and ligaments (Posthumus et al., 2011b; Brown et al., 2011b). Collagen type $\mathrm{V}$ is a protein encoded by the COL5A1 gene which is located on the long (q) arm of chromosome 9 (Collins and Posthumus,
2011). Mutations within the COL5A1 gene cause the classic form of Ehlers-Danlos syndrome characterized by joint hyper mobility (Brown et al., 2011) and other joint dysfunctions (Collins and Posthumus, 2011). Therefore, our attention was directed toward COL5A1 sequence variants as potential intrinsic risk factors for the ACL rupture.

The COL5A1 gene contains BstUI and DpnII restriction fragment length polymorphisms (RFLPs) within its 3'-untranslated region (UTR) (Greenspan and Pasquinelli, 1994). The functional COL5A1 Sp1 binding site polymorphism - BstUI RFLP, known also as: rs $12722, \mathrm{C} / \mathrm{T}$ is associated with a number of sport injuries. This polymorphism was detected in a physically active Caucasian population in South Africa (Posthumus et al., 2010).

The CC genotype of this variant was overrepresented significantly in the asymptomatic control participants of the study. The CC genotype of SNP rs 12722 was also overrepresented in the asymptomatic control participants among a physically active Caucasian population in Australia. The individuals with a wild-type CC genotype are protected against chronic degenerative changes in the Achilles tendon during running and other forms of physical activity (Collins and Posthumus, 2011). Similar results were reported when female participants with ACL ruptures were compared with asymptomatic female controls. This data suggests that individuals with a CC genotype are protected, despite the load or external forces applied to their musculoskeletal soft tissues. Another polymorphism which we investigated was DpnII RFLP, known also as rs 13946, C/T within the COL5A1 gene. A Mokone et al. (2006) study revealed a significant difference in the allele frequencies of the COL5A1 BstUI RFLP between the Achilles tendon pathology group and control subjects. However, the influence of both polymorphisms on the expression of the COL5A1 gene is not completely known.

The aim of this study was to examine the association of the BstUI RFLP and DpnII RFLP polymorphisms in the COL5A1 gene, individually and as haplotypes, with ACL ruptures in recreational skiers. We postulated that the COL5A1 BstUI RFLP and COL5A1 DpnII RFLP polymorphisms would be individually associated 
with the ACL rupture and that interaction between the COL5A1 BstUI RFLP and COL5A1 DpnII RFLP polymorphisms as a haplotype would predispose recreational skiers to higher risk of the ACL rupture.

\section{Material and Methods}

The Pomeranian Medical University Ethics Committee approved the study and written informed consent was obtained from each participant. The study complied with the guidelines set out in the ethics policy of the Szczecin University (Kruk, 2013).

A total of 138 male recreational skiers (27 \pm 2 years) with surgically diagnosed primary ACL ruptures were recruited for this study, all of whom qualified for ligament reconstruction. The control group was comprised of 183 apparently healthy male skiers (26 \pm 3 years) with a comparable level of exposure to ACL injury, none of whom had any self-reported history of ligament or tendon injury.

We followed recent recommendations for genotype-phenotype association studies (Chanock et al., 2007; Attia et al., 2009). Genomic DNA was extracted from the oral epithelial cells using GenElute Mammalian Genomic DNA Miniprep Kit (Sigma, Germany) according to the manufacturer's protocol. Allelic discrimination of COL5A1 BstUI RFLP C/T (rs 12722) and DpnII RFLP C/T (rs 13946) polymorphic sites was performed using a TaqMan Pre-Designed SNP Genotyping Assays (Applied Biosystems, USA), including primers and fluorescently labelled (FAM and VIC) probes for the detection of alleles. All samples were genotyped on a real-time polymerase chain reaction (PCR) instrument (StepOne ${ }^{\mathrm{TM}}$, Applied Biosystems, USA). Thermal cycler conditions were as follows: an initial step at $95^{\circ} \mathrm{C}$ for $5 \mathrm{~min}$, followed by 45 cycles of denaturation at $94^{\circ} \mathrm{C}$ for $15 \mathrm{~s}$ and anneal/extend at $60^{\circ} \mathrm{C}$ for $1 \mathrm{~min}$. We used positive and negative controls for the detection of both polymorphisms. The results were gathered by two experienced and independent investigators who were blind to the participants' data.

\section{Statistical analysis}

Some differences in the genotypes and allele frequency were analyzed using ${ }^{2}$ or Fisher exact tests. Allelic-based odds ratios (OR) with 95\% confidence intervals $(95 \% \mathrm{CI})$ were calculated using logistic regression analysis. A post hoc power calculation was detected for each gene variant. The genotypes between cases and controls were compared in three ways. First, a general test of association in the 2-by-3 table of phenotype-by-genotype was performed. Then two different modes of inheritance of minor allele were assumed: dominant, in which homozygotes and heterozygotes for the minor allele were pooled and compared to homozygotes for the major allele, and recessive, in which homozygotes for the minor allele were compared to pooled homozygotes and heterozygotes for the major allele. In addition, the programming language and environment $\mathrm{R}$ (http://www.r-project.org) were used for Hardy-Weinberg, linkage disequilibrium (LD) testing (package genetics), for the haplotype analysis (package haplo.stats). The haplo.em, haplo.group, and haplo.score functions of the haplo.stats package were used to infer haplotype frequencies and to test the association between reconstructed haplotypes and the risk of the ACL rupture assuming three possible haplotype effects: additive, dominant and recessive. Hap.score is the statistical score for haplotypes reflecting the strength of association; the positive value of hap.score indicates increased risk of ACL injury for a particular haplotype, while the negative value indicates reduced risk. The pair-wise linkage disequilibrium between BstUI RFLP C/T and DpnII RFLP $\mathrm{C} / \mathrm{T}$ was estimated by $\mathrm{D}^{\prime}$ and $\mathrm{r}^{2}$. For all tests, the level of significance was set at $\mathrm{p}<0.05$.

\section{Results}

At baseline, male recreational skiers with surgically diagnosed primary ACL ruptures and the control group did not differ significantly by age (27 \pm 2 and $26 \pm 3$ years, respectively), and the level of exposure to ACL injury. The genotype and allele frequencies for the COL5A1 BstUI RFLP $\mathrm{C} / \mathrm{T}$ (rs12722) and COL5A1 DpnII RFLP C/T (rs13946) are shown in Table 1. The genotype distributions for both polymorphisms met HardyWeinberg expectations in both groups.

There were no significant differences in genotype distribution or allele frequency of either COL5A1 BstUI RFLP (rs12722, C/T) or COL5A1 DpnII RFLP (rs13946, C/T) polymorphisms between the ACL rupture group and the control group using the 2-by-3 general test of association 
(Table 1). Likewise, there were no significant differences in the genotype frequencies for the COL5A1 BstUI RFLP C/T and DpnII RFLP C/T polymorphisms, when dominant and recessive modes of inheritance were assumed.

COL5A1 BstUI RFLP C/T and DpnII RFLP C/T were found to be in linkage disequilibrium (Table 2). A total of three reconstituted haplotypes with estimated frequency $>0.05$ were found, and only those were evaluated for an association with the ACL rupture. The T-T (BstUI RFLP T, DpnII RFLP $\mathrm{T})$ haplotype was the most common $(55.5 \%)$. The haplotype T-C was not present in any of the subjects.
Then, we tested the association between these haplotypes and the ACL rupture assuming three haplotype effects; a) additive - considering the count of a particular haplotype as $0,1,2, \mathrm{~b}$ ) dominant - heterozygous or homozygous carrier of a particular haplotype versus otherwise, c) recessive - homozygous for a particular haplotype versus otherwise. None of these yielded statistically significant results, however there was an underrepresentation tendency of the C-T haplotype in the ACLR group compared to controls under the recessive mode of inheritance (Table 3).

Table 1

Association between the presence of either COL5A1 BstUI RFLP (rs12722, C/T) \begin{tabular}{cccccc} 
or COL5A1 DpnII RFLP (rs13946, C/T) polymorphisms and incidence of ACL rupture \\
\hline SNP Group HWE Genotype $p$ & $p$ & Allele $p$ & OR $(95 \% \mathrm{CI})$
\end{tabular} distribution frequency

\begin{tabular}{llllllll}
\hline BstUI & ACLR & 0.861 & CC: $24(17 \%)$ & p 0.467 & T: $59 \%$ & 0.218 & $0.82(0.59-1.14)$ \\
RFLP C/T & $\mathrm{n}=138$ & & CT: $66(48 \%)$ & pD 0.276 & C: $41 \%$ & & \\
& & & TT: $48(35 \%)$ & pR 0.398 & & &
\end{tabular}

\begin{tabular}{|c|c|c|c|}
\hline \multirow{3}{*}{$\begin{array}{c}\text { Control } \\
n=183\end{array}$} & 1 & CC: $39(21 \%)$ & $\mathrm{T}: 54 \%$ \\
\hline & & CT: $91(50 \%)$ & C: $46 \%$ \\
\hline & & TT: $53(29 \%)$ & \\
\hline
\end{tabular}

\begin{tabular}{|c|c|c|c|c|c|c|c|}
\hline DpnII & ACLR & 1 & CC: $12(9 \%)$ & p 0.393 & $\mathrm{~T}: 71 \%$ & 0.846 & $0.97(0.68-1.38)$ \\
\hline RFLP & $\mathrm{n}=138$ & & CT: $57(41 \%)$ & pD 0.499 & C: $29 \%$ & & \\
\hline \multirow[t]{4}{*}{$\mathrm{C} / \mathrm{T}$} & & & TT: $69(50 \%)$ & pR 0.387 & & & \\
\hline & Control & 0.077 & CC: $11(6 \%)$ & & $\mathrm{T}: 70 \%$ & & \\
\hline & & & CT: $88(48 \%)$ & & C: $30 \%$ & & \\
\hline & $\mathrm{n}=183$ & & TT: $84(46 \%)$ & & & & \\
\hline
\end{tabular}

$p$ values correspond to genotype distribution and allele frequency.

OR correspond to the odds ratio for the incidence of ACL rupture.

ACLR: anterior cruciate ligament rupture; HWE: Hardy-Weinberg Equilibrium;

OR: odds ratio; CI: confident interval, $p_{D}$ and $p_{R}$ are two-sided Fisher's

exact test probabilities for dominant $(C C+C T$ vs TT) and recessive (CC+CT vs TT) modes of inheritance of the minor allele (BstUI RFLP C/T, DpnII RFLP C/T), respectively. 
Table 2

Pair-wise linkage disequilibrium $(L D)$ and inferred haplotype frequencies for COL5A1 BstUI RFLP C/T and DpnII RFLP C/T

\begin{tabular}{lcccccc} 
& & & & & \multicolumn{2}{c}{ Haplotypes } \\
\cline { 5 - 7 } Gene & SNP1 & SNP2 & $\mathrm{D}$ & $\mathrm{r}^{2}$ & Haplotype & $\begin{array}{c}\text { Frequency } \\
(\%)\end{array}$ \\
\hline COL5A1 & $\begin{array}{c}\text { BstUI RFLP C/T } \\
(\text { rs12722) }\end{array}$ & $\begin{array}{c}\text { DpnII RFLP C/T } \\
(\text { rs13946) }\end{array}$ & $0.98^{+}$ & 0.52 & T-T & 56
\end{tabular}

$(\mathrm{rs} 12722)$

(rs13946)

C-C

29

C-T $\quad 15$

$\mathrm{T}-\mathrm{C} \quad 0.0$

Total

100.0

$D^{\prime}$ - the deviation of the observed frequency of a haplotype from the expected.

$D^{\prime}=0.98$ suggest on very high LD between COL5A1 BstUI RFLP C/T and COL5A1 DpnII RFLP C/T.

Haplotypes are in decreasing frequency.

${ }^{+} p<0.0001$

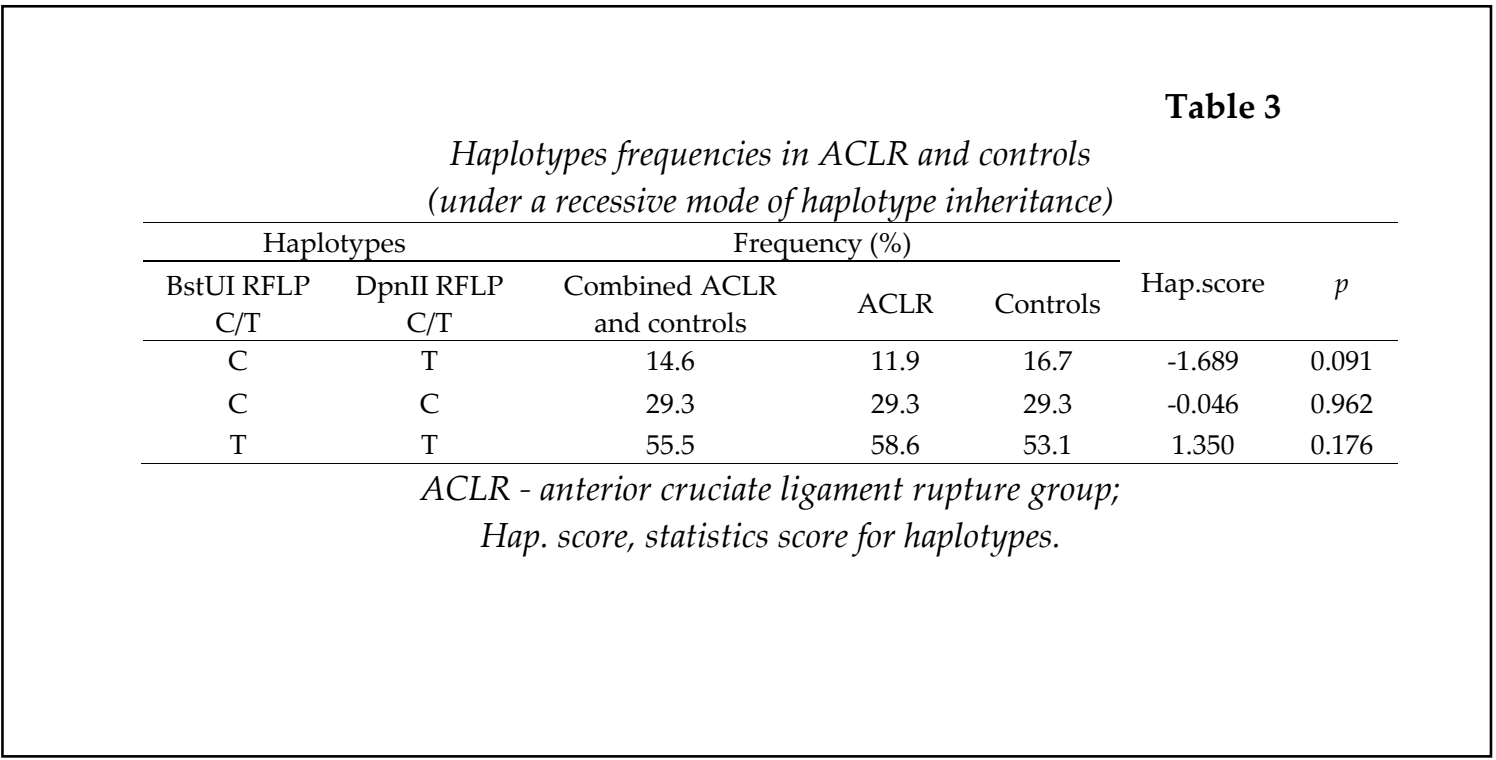

\section{Discussion}

The biological mechanisms involved in noncontact musculoskeletal soft tissue injuries are poorly understood, but genetic risk factors may be associated with susceptibility to injuries. A single nucleotide polymorphism - DNA sequence variation can occur throughout the genome and can also affect the response of an individual to a specific treatment or other stimuli (Pruna et al., 2013). Smith et al. (2012) reported familiar predisposition to non-contact ACL tears. The genetic influence was assessed among others with a medical history questionnaire to determine the knee ligament injuries of the patients' primary family members. There was a higher incidence 
rate of ACL injury in the immediate family of the injured group (35\%) than that of the control group $(4 \%)$.

In our study, we examined the association between both BstUI RFLP C/T (rs12722) and DpnII RFLP C/T (rs13946) polymorphisms in the COL5A1 gene, individually and as haplotypes, with anterior cruciate ligament ruptures in recreational Polish skiers. Our findings showed no significant differences in genotype distribution or allele frequencies of COL5A1 BstUI RFLP C/T and COL5A1 DpnII RFLP C/T polymorphisms between the ACL rupture group and control group. Thus, our initial hypothesis was not confirmed. However, we found an underrepresentation tendency of the C-T haplotype in the ACLR group compared to controls $(\mathrm{p}=0.091)$. Higher frequency of the COL5A1 C-T (BstUI RFLP C/T and DpnII RFLP $\mathrm{C} / \mathrm{T}$ polymorphisms) haplotype among a group of apparently healthy male recreational skiers suggests an association with reduced risk of anterior cruciate ligament injury.

The results of the Smith et al. (2012) study helped to identify genetic factors associated with ACL tears. This study was based on unmatched case-control designs and was performed on a Caucasian South African population. The COL1A1 gene encodes a protein chain within type I collagen, a major structural component of ligaments. A rare TT genotype of the COL1A1 Sp1 binding site polymorphism was underrepresented in those with ACL injuries in comparison with the controls. ACL-injured subjects were 4 times more likely to have a family member with a ligament injury of any kind in comparison with controls. Another COL12A1 gene encodes for protein chains in type XII collagen, which is believed to regulate fibril diameter in ligaments. The researchers found that the AA genotype of the COL12A1 AluI polymorphism was overrepresented in female subjects with ACL injury. Recently, an association between the chromosomal region 11q22 and risk of the ACL tear was reported (Posthumus et al., 2012). Several matrix metalloproteinase genes, including physiologic mediators of collagen cleavage and removal, are located on the chromosome 11q22. In ACL-injured subjects, AG and GG genotypes of 1 matrix metalloproteinase variant were significantly underrepresented when compared to uninjured subjects. The frequency of these variant haplotypes within the gene was significantly different between injured and uninjured groups.

Likewise, the CC genotype of a variant in the COL5A1 gene has been associated with ACL tears in females (Collins and Posthumus, 2011). The CC genotype was overrepresented in the asymptomatic control participants among a physically active Caucasian population in Australia. On the other hand, individuals with a wild-type CC genotype are protected against chronic degenerative changes in the Achilles tendon during running and other physical activity (O'Connell et al., 2013). O'Connell et al. (2013) support the hypothesis that variants within genes, such as COL5A1, COL3A1, COL6A1, and COL12A1 genes that code for connective tissue components of the musculoskeletal system, may modulate susceptibility to exercise-associated muscle cramping (EAMC). The aim of mentioned study was to investigate a potential association between COL5A1 rs12722 (C/T), COL3A1 rs1800255 (G/A), COL6A1 rs35796750 (T/C), and COL12A1 rs970547 (A/G) polymorphisms and a history of EAMC. The COL5A1 CC genotype was significantly overrepresented among the group without EAMC $(22 \%)$ when compared with the EAMC group $(11 \%)$. Thus, the COL5A1 gene as a potential marker for a history of EAMC was identified for the first time. The COL5A1 (the variant rs12722, BstUI RFLP) may also be a candidate gene associated with the development of the bilateral quadriceps tendon rupture (Longo et al., 2010). A polymorphism (rs12722) within the functional COL5A1 3'-untranslated region (UTR) has been shown to associate with chronic Achilles tendinopathy and other exercise-related phenotypes (Abrahams et al., 2013). This investigation included over 600 Caucasians participants who were genotyped for the COL5A1 3'-UTR markers rs71746744, rs16399, rs1134170 and marker rs4919510 within MIR608. All genetic markers were independently associated with chronic Achilles tendinopathy.

Studies by Raleigh et al. (2009) showed variants within the MMP3 gene were associated with Achilles tendinopathy. There were significant associations between the GG genotype of rs679620, the CC genotype of rs591058, the AA genotype of rs650108 and risk of Achilles tendinopathy. The ATG haplotype (rs679620, 
rs591058, and rs650108) was underrepresented in the tendinopathy group when compared to the control group.

A strength of our study is the homogeneity of the investigated groups. However, this fact may also be considered as a limitation, because only male skiers were examined. Other studies suggest that the rate of the ACL rupture is even three times higher in female than in male athletes (Warden et al., 2006). Female athletes landing with inadequate knee flexion, in greater-than-normal valgus, and external rotation are at increased risk of ACL injury when compared to men participating in similar athletic activities (Hewett et al., 2010; Sutton, 2013). Intrinsic factors such as an increased quadriceps angle, an increased posterior tibial slope, and estrogen fluctuation may also predispose females to ACL injury (Warden et al., 2006).

The importance of genetics in sport increases every year. Studies of the genetic factors that influence the risk of injuries are still scarce, even though they may provide a useful tool for the sports selection process, individualization of the training programs or sports traumatology. The results of the present study indicate that modifying training loads or physical therapy may be used as preventive means in athletes that are genetically predisposed to ACL injury.

\section{Conclusions for competitive sports}

The findings of the present study provide a better understanding of which genetic profiles contribute to higher ACL injury risk.

We indicated that there was an underrepresentation tendency of the C-T haplotype in the ACLR group when compared to controls $(\mathrm{p}=0.091)$. Higher frequency of the COL5A1 C-T (BstUI RFLP C/T and DpnII RFLP $\mathrm{C} / \mathrm{T}$ polymorphisms) haplotype among a group of apparently healthy male skiers suggests an association with reduced risk of anterior cruciate ligament injury.

Analysis of the complex relationship between gene variants and ACL ruptures among athletes may assist clinicians and athletes to optimize training and to reduce the risk of the $\mathrm{ACL}$ rupture.

\section{References}

Abrahams Y, Laguette MJ, Prince S, Collins M. Polymorphisms within the COL5A1 3'-UTR that alters mRNA structure and the MIR608 gene are associated with Achilles tendinopathy. Ann Hum Genet., 2013; 77(3): 204-14

Attia J, Ioannidis JP, Thakkinstian A. How to use an article about genetic association: B: Are the results of the study valid? J Am Med Assoc., 2009; 301(2): 191-197

Bere T, Mok KM, Koga H, Krosshaug T, Nordsletten L, Bahr R. Kinematics of anterior cruciate ligament ruptures in world cup alpine skiing: 2 case reports of the slip-catch mechanism. Am J Sports Med., 2013; 41(5): 1067-73

Boyan BD, Hart DA, Enoka RM, Nicolella DP, Resnick E, Berkley KJ, Sluka KA, Kwoh CK, Tosi LL, O'Connor MI, Coutts RD, Kohrt WM. Hormonal modulation of connective tissue homeostasis and sex differences in risk for osteoarthritis of the knee. Biol Sex Differ., 2013; 4(1): 3

Brown JC, Miller CJ, Schwellnus MP, Collins M. Range of motion measurements diverge with increasing age for COL5A1 genotypes. Scand J Med Sci Sports., 2011; 21: e266-e272

Chanock SJ, Manolio T, Boehnke M. Replicating genotype - phenotype associations. Nature., 2007; 447(7145): 655-660

Collins M, Posthumus M. Type V Collagen Genotype and Exercise-Related Phenotype Relationships: A Novel Hypothesis. Exercise and Sport Sciences Reviews, 2011; 39(4): 191-8

Dhillon MS, Bali K, Prabhakar S. Proprioception in anterior cruciate ligament deficient knees and its relevance in anterior cruciate ligament reconstruction Indian. J Orthop., 2011; 45(4): 294-300 
Frank CB. Ligament structure, physiology and function. J Musculoskelet Neuronal Interact., 2004; 4: 199-201

Frizziero A, Ferrari R, Giannotti E, Ferroni C, Poli P, Masiero S. The meniscus tear. State of the art of rehabilitation protocols related to surgical procedures. Muscles, Ligaments and Tendons Journal, 2012; 2(4): 295-301

Greenspan DS, Pasquinelli AE. BstUI and DpnII RFLPs at the COL5A1 gene. 1. Hum Mol Genet., 1994; 3: 385

Hasler RM, Benz J, Benneker LM, Kleim B, Dubler S, Zimmermann H, Exadaktylos AK. Do alpine skiers and snowboarders wear protective equipment more often after an accident? Swiss Med Wkly., 2011; 141: w13283

Hewett TE, Ford KR, Hoogenboom BJ, Myer GD. Understanding and preventing ACL injuries: current biomechanical and epidemiologic considerations - update 2010, N Am J Sports Phys Ther., 2010; 5(4): 234-251

Hewett TE, Lynch TR, Myer GD. Multip-le risk factors related to familial predisposition to anterior cruciate ligament injury: fraternal twin sisters with anterior cruciate ligament ruptures. Br J Sports Med., 2010; 44(12): 848-855

Kruk J. Good scientific practice and ethical principles in scientific research and higher education. Central European Journal of Sport Sciences and Medicine, 2013; Vol. 1 No. 1/2013: 25-29

Longo UG, Fazio V, Poeta ML, Rabitti C, Franceschi F, Maffulli N, Denaro V. Bilateral consecutive rupture of the quadriceps tendon in a man with BstUI polymorphism of the COL5A1 gene. Knee Surg Sports Traumatol Arthrosc., 2010; 18(4): 514-8

Mokone GG, Schwellnus MP, Noakes TD, Collins M. The COL5A1 gene and Achilles tendon pathology. Scand J Med Sci Sports., 2006; 16(1): 19-26

Myer GD, Brent JL, Ford KR, Hewett TE. Real-time assessment and neuromuscular training feedback techniques to prevent ACL injury in female athletes. Strength Cond J., 2011; 1; 33(3): 21-35

O'Connel K, Saunders CJ, Collins M. Collagen gene sequence variants in exercise-related traits. Central European Journal of Sport Sciences and Medicine., 2013; Vol. 1 No. 1/2013: 3-17

O'Connell K, Posthumus M, Schwellnus MP, Collins M. Collagen genes and exercise-associated muscle cramping. Clin J Sport Med., 2013; 23(1): 64-9

O'Donnell ME, Badger SA, Campbell D, Loan W, Sinnott B. The skiers knee without swelling or instability, a difficult diagnosis: a case report. J Med Case Reports., 2007; 1: 11

Posthumus M, Schwellnus MP, Collins M. The COL5A1 Gene: A Novel Marker of Endurance Running Performance. Medicine E Science In Sports \& Exercise, 2011

Posthumus M, September AV, Schwellnus MP, Collins M. The COL5A1 gene and musculoskeletal soft-tissue injuries. SAJSM, 2010; vol 22, No. 2

Posthumus M, Collins M, van der Merwe L, O'Cuinneagain D, van der Merwe W, Ribbans WJ, Schwellnus MP, Raleigh SM. Matrix metalloproteinase genes on chromosome 11q22 and the risk of anterior cruciate ligament (ACL) rupture. Scand J Med Sci Sports., 2012; 22(4): 523-33

Pruna R, Artells R, Ribas J, Montoro B, Cos F, Muñoz C, Rodas G, Maffulli N. Single nucleotide polymorphisms associated with non-contact soft tissue injuries in elite professional soccer players: influence on degree of injury and recovery time. BMC Musculoskelet Disord., 2013; 14: 221

Raleigh SM, van der Merwe L, Ribbans WJ, Smith RK, Schwellnus MP, Collins M. Variants within the MMP3 gene are associated with Achilles tendinopathy: possible interaction with the COL5A1 gene. Br J Sports Med., 2009; 43(7): 514-20

Sawczuk M, Maciejewska A, Cięszczyk P, Eider J. The role of genetic research in sport. Sci. Sports., 2011; 26: 251-258

Smith HC, Vacek P, Johnson RJ, Slauterbeck JR, Hashemi J, Shultz S, Beynnon BD. Risk Factors for Anterior 
Cruciate Ligament Injury. A Review of the Literature-Part 2: Hormonal, Genetic, Cognitive Function, Previous Injury, and Extrinsic Risk Factors. Sports Health., 2012; 4(2): 155-161

Spörri J, Kröll J, Schwameder H, Schiefermüller Ch, Müller E. Course setting and selected biomechanical variables related to injury risk in alpine ski racing: an explorative case study. Br J Sports Med., 2012; 46(15): 1072-1077

Sutton KM, Bullock JM. Anterior cruciate ligament rupture: differences between males and females. J Am Acad Orthop Surg., 2013; 21(1): 41-50

Tovar N, Bourke S, Jaffe M, Murthy NS, Kohn J, Gatt C, Dunn MGA. Comparison of degradable synthetic polymer fibers for anterior cruciate ligament reconstruction. J Biomed Mater Res A., 2010; 93(2): 738-747

Warden SJ, Saxon LK, Castillo AB, Turner CH. Knee ligament mechanical properties are not influenced by estrogen or its receptors. Translational physiology, Am J Physiol., 2006; Vol. 290 no. E1034-E1040

\section{Corresponding author:}

\section{Paweł Cięszczyk, PhD}

University of Szczecin, Department of Physical Culture and Health Promotion, Poland

Al. Piastów 40B blok 6, 71-065 Szczecin

Phone/Fax: +48 914442735

E-mail: cieszczyk@poczta.onet.pl 